\title{
A 72-year-old man with intermittent fever, anemia and a history of coronary and peripheral artery disease
}

\author{
Stefano Del Pace $\cdot$ Andrea Savino $\cdot$ Raffaele Rasoini $\cdot$ Camilla Alderighi $\cdot$ \\ Manlio Acquafresca - Alessandro Alessi Innocenti - Carlo Pratesi • \\ Gian Franco Gensini
}

Received: 1 April 2010/Accepted: 27 April 2010/Published online: 5 June 2010

(c) SIMI 2010

\section{Case presentation}

Dr. Del Pace: A 72-year-old man with history of coronary and peripheral artery disease was admitted to this hospital with fever, fatigue and loss of weight. He had hypertension, type 2 diabetes mellitus and hyperlipidemia. He suffered from an ischemic cardiomyopathy with a low ejection fraction $(33 \%)$. Fifty years prior he had undergone subtotal gastroresection for peptic ulcer. Seventeen years prior to this current admission (PTA), he had an aortobifemoral bypass grafting for symptomatic peripheral artery disease, and 8 years prior he had a myocardial infarction. Three vessel coronary disease was detected and coronary-artery bypass grafting had been carried out.

One year PTA, he experienced an episode of sustained ventricular tachycardia, treated with stenting of the anterior descending coronary artery, followed by an internal cardiac defibrillator (ICD) implantation. Ten months PTA, he had a massive enteric bleeding complicated by hemorrhagic

S. Del Pace · A. Savino ( $₫) \cdot$ G. F. Gensini

Department of Heart and Vessels, Careggi Hospital,

University of Florence, Viale Morgagni 85,

50139 Florence, Italy

e-mail: savino.andrea@gmail.com

R. Rasoini · C. Alderighi

Istituto Fiorentino di Cura e Assistenza, Florence, Italy

M. Acquafresca

Radiology Service, Department of Diagnostic Images, Careggi Hospital, University of Florence, Florence, Italy

A. A. Innocenti · C. Pratesi

Department of Medical and Surgical Critical Care,

Careggi Hospital, University of Florence, Florence, Italy shock. A colonoscopy revealed ulcerated cecal angiodysplasia that was treated with an application of metallic clips.

During the following months, he was admitted several times for sideropenic anemia without evidence of acute gastrointestinal bleeding, and iron therapy was administrated. Two colonoscopies were performed but no source of active bleeding was detected.

Two months PTA, he developed a low-grade fever resistent to a 2-week course of antibiotic therapy with levofloxacin.

Laboratory tests showed a normocytic anemia $(8.1 \mathrm{~g} / \mathrm{dL})$ with normal levels of B12 and folic acid, with low levels of sideremia (13 $\mu \mathrm{g} / \mathrm{dL})$, and a normal haptoglobin.

An esophagogastroduodenoscopy was normal. Neoplastic markers (NSE, CA 125, CA 15-3, CA 72-4, CA 19-9, CYFRA 21-1, CEA, alfa-FP) were all in the normal range. Antinuclear antibody, ANCA, rheumatoid factor were negative as well as immunofixation in serum and urine.

A computed tomography (CT scan) of the abdomen with contrast material confirmed ceacum angiodysplasia, but did not reveal active enteral bleeding. Transesophageal echocardiography was performed, and a vegetation on the ICD catheter was found. One blood culture was positive for Streptococcus intermedius. Treatment with amoxicillin was started, and during the hospital stay, the fever disappeared. Removal of the pacemaker was suggested, but the patient refused it. After discharge, he completed a 4-week cycle of antibiotic therapy, but after 1 week of withdrawal, fever developed again with malaise, fatigue and weight loss. He was readmitted to this hospital.

On examination, the patient appeared in mild distress. He had low-grade fever $\left(37.5^{\circ} \mathrm{C}\right)$. The Blood pressure was $100 / 50 \mathrm{mmHg}$, pulse rate 70 beats/min and oxygen saturation $98 \%$ while he was breathing ambient air. A grade 2/6 
systolic murmur was heard at the left sternal border. The site of the ICD was nonerythematous, but tender on palpation; fine crackles were heard at both lung bases; the legs were warm without pitting edema, with weak pulses. The abdomen was soft and non-tender, with normal bowel sounds. No hepatosplenomegaly were present. The remainder of the physical examination was unremarkable.

An electrocardiogram showed a sinus rhythm and nonspecific anomalies of ventricular repolarization. Laboratory tests were performed (Table 1). Two units of packed red cells were administered. The intermittent fever persisted, but several blood cultures were negative. Fecal occult blood tests were negative. A transthoracic echocardiogram showed a dilatated left ventricle with a depressed ejection fraction (33\%). A transesophageal echocardiogram confirmed the vegetation previously detected on the pacemaker catheter. We again proposed the removal of pacemaker, and this time the patient accepted; he underwent a successful procedure. Cultures of the catheter tip were positive for $S$. intermedius. Specific therapy with intravenous teicoplanin and levofloxacin was administered.

After a 2-week course of intravenous antibiotic therapy, fever and malaise persisted with low levels of hemoglobin $(8.9 \mathrm{~g} / \mathrm{dL})$ and high levels of CRP $(57 \mathrm{mg} / \mathrm{dL})$.

\section{Differential diagnosis}

Dr. Rasoini, Dr. Savino: A common cause of fever of uncertain origin is infective endocarditis. Pacemaker infection occurs in $0.13-19.9 \%$ of patients with inserted pacemakers, and catheter vegetations are detectable in $10 \%$ of the infections linked to the presence of the device. Our patient presented with fever, chills, anemia, fatigue, loss of weight that are all consistent with endocarditis. A blood culture was positive for $S$. intermedius, one of the most common pathogen involved in infective endocarditis (although not typical for pacemaker endocarditis). A transesophageal echocardiogram and culture of the catheter confirmed the diagnosis, and excluded other cardiac sites of infection, but fever persisted and markers of inflammation remained high despite ICD removal and antibiotic therapy. Moreover, we had some concerns about the severe worsening anemia that endocarditis could hardly justify.

Angiodysplasias are arteriovenous malformations that are common causes of lower gastrointestinal bleeding in elderly patients. Bleeding caused by these lesions can be chronic, slow and intermittent, or massive and acute. They can present with overt hemorrhage, or insidiously with iron deficiency anemia. Bleeding stops spontaneously in 85$90 \%$ of cases, but it recurs in $25-85 \%$ of cases. The patient had a demonstrated cecal angiodysplasia that led to
Table 1 Laboratory data

\begin{tabular}{ll}
\hline Variables & On admission \\
\hline Hemoglobin & $6.3 \mathrm{~g} / \mathrm{dL}$ \\
Hematocrit & $19.9 \%$ \\
Red cell count & $2,470 \times 10^{3} / \mathrm{mm}^{3}$ \\
Mean corpuscular hemoglobin concentration & $31.7 \%$ \\
Reticulocytes & $2.02 \%$ \\
White-cell count & $6,730 / \mathrm{mm}^{3}$ \\
Differential count $(\%)$ & \\
Neutrophils & 83.1 \\
Lymphocytes & 7.9 \\
Monocytes & 8.2 \\
Eosinophils & 0.7 \\
Basophils & 0.1 \\
Platelet count & $214,000 / \mathrm{mm}$ \\
Procalcitonin & $0.19 \mathrm{mg} / \mathrm{L}$ \\
Haptoglobin & $2.87 \mathrm{mg} / \mathrm{dL}$ \\
Total bilirubin & $0.45 \mathrm{mg} / \mathrm{dL}$ \\
Erythrocyte sedimentation rate & $32 \mathrm{~mm} / \mathrm{h}$ \\
C-reactive protein & $28 \mathrm{mg} / \mathrm{L}$ \\
Iron & $206 \mu \mathrm{g} / \mathrm{dL}$ \\
Ferritin & $106 \mathrm{ng} / \mathrm{mL}$ \\
Folic acid & $37.62 \mathrm{ng} / \mathrm{mL}$ \\
Vitamin B12 & $188 \mathrm{pg} / \mathrm{mL}$ \\
Urine analysis & Normal \\
\hline & \\
&
\end{tabular}

massive gastroenteric bleeding 10 months PTA, (while the patient was on dual antiplatelet therapy because of the recent coronary stenting).

Cecal angiodysplasia could explain the chronic bleeding with worsening anemia, but is not easily related to the inflammation signs of our patient.

In a patient with anemia due to gastroenteric bleeding and with a prior history of gastrectomy, it is mandatory to rule out a gastric cancer or another enteric neoplasm. Nevertheless, neoplastic markers were in the normal range, and no mucosal defects were detected during esophagogastroduodenoscopy. Furthermore, colonoscopy was normal (except for the cecal angiodysplasia) and a CT scan of the abdomen did not reveal any abnormality of the stomach wall nor any other enteric mass.

Inflammatory bowel diseases present more frequently between 15 and 30 years of age, but late presentations are also described. Weight loss, anorexia and fever are common. Elevated levels of C-reactive protein, erythrocyte sedimentation rate, as well as anemia, are frequent laboratory findings. Nonetheless, the patient had never complained of abdominal pain or diarrhea, which are very common signs of these disorders, and neither colonoscopy nor a CT scan of the abdomen revealed findings suggestive of inflammatory bowel disease. 
Ischemic colitis should be considered in a patient with coronary and peripheral artery disease. The patient did not complain of abdominal pain related to food consumption. The angio-TC examination of the abdomen was non supportive for the diagnosis. Moreover, colonoscopy did not show edema, erythema, submucosal hemorrhage, or partial mucosal necrosis with ulceration, and signs of inflammation would not be expected in the clinical presentation.

Aortoenteric fistulas with a periprosthetic infection could have an endocarditis-like presentation (i.e., anemia, fever, fatigue, loss of weight) because of local and systemic infection and anemia due to intermittent blood loss and systemic inflammation. Endocarditis can also be secondary to septic embolisation from the fistula.

Aortoenteric fistulas have been described even 10 years after implantation of aortobifemoral bypass graft.

This kind of fistula is hard to detect on imaging because bleeding is often intermittent, so that fecal occult blood tests are often negative.

In addition, in our patient, fever did not disappear and inflammatory markers remained high even after removal of the ICD and antibiotic therapy, without a definite source of infection or inflammation: these findings could be related to a periprosthetic infective process.

\section{Further investigations}

Dr. Alderighi: Fecal occult blood tests were persistently negative. A 99mTc-labeled red blood cell showed a minimal activity in the right iliac fossa compatible with colon angiodysplasia. Indium-labeled white-cell whole-body study showed a pathologic accumulation under the liver and located in front of the right kidney, corresponding to the third part of the duodenum. A whole-body positron emission tomography found a pathologic fluid collection under the liver, corresponding to the pathologic white-cell SPECT findings. The patient underwent a blood transfusion for a new anemia (hemoglobin $7.1 \mathrm{~g} / \mathrm{dL}$ ). A fecal occult blood test performed in this circumstance was positive. A new CT scan guided by PET findings, performed with the administration of contrast medium, demonstrated a close association of the proximal limbs of the aortobifemoral bypass graft with the third part of duodenum. It revealed also an enlargement of the tissue mass surrounding the proximal limbs of the bypass extending for $2 \mathrm{~cm}$, with mild enhancement after administration of the contrast material, and an air-fluid collection, suggesting a periprosthetic infective process (Fig. 1).

Prof. Pratesi, Dr. Innocenti: The patient was taken to the operating room. A periprosthetic enteric fistula was found between the third part of the duodenum and the

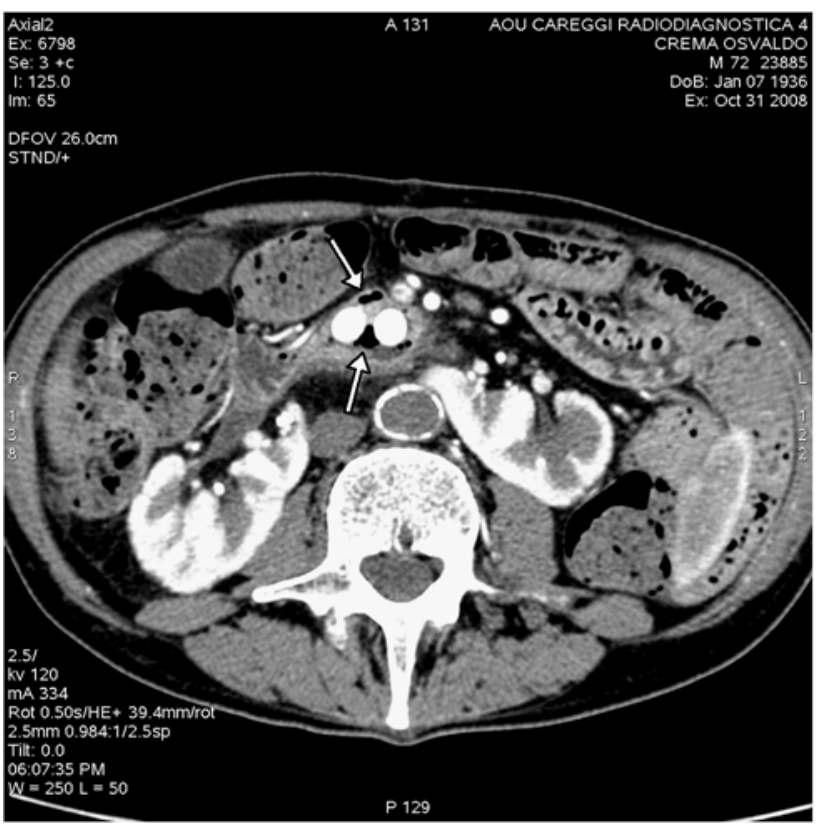

Fig. 1 Ectopic gas (white arrows) in the context of the soft tissue around the proximal limbs of the bypass

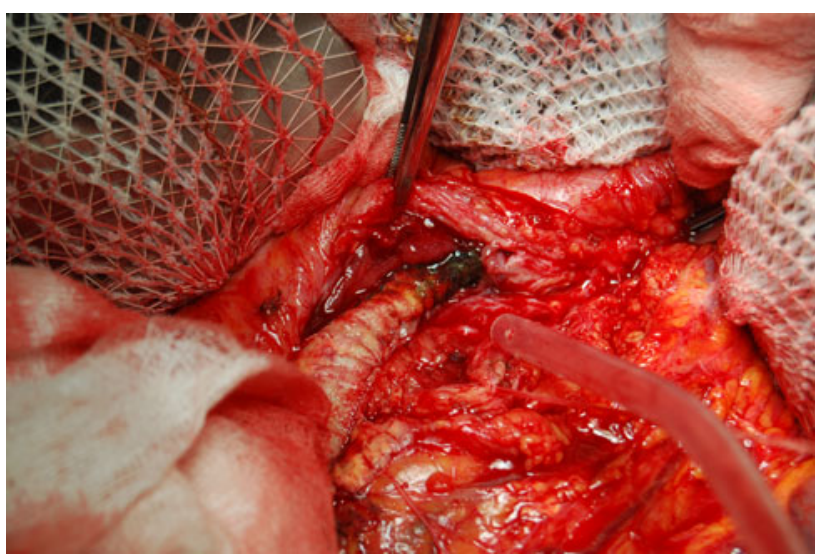

Fig. 2 The aortobifemoral by pass graft in contact with the duodenum that has been lifted up

proximal right limb of the aortobifemoral bypass graft. A broad damage to the duodenum was found, as well as bile staining and erosion of the proximal right limb of the graft (Fig. 2). No overt exudate was seen at the site, but the graft was noted to be very densely adherent to the surrounding tissues. The aortobifemoral bypass graft was removed, and the defect of the duodenum was repaired. An axillo-bifemoral bypass with subcutaneous tunneling was performed (Fig. 3).

The postoperative course was characterized by respiratory distress from bilateral pneumonia followed by cardiopulmonary arrest from ventricular tachycardia, and the patient underwent advanced cardiac life support and broadspectrum antibiotic therapy. Clinical conditions improved 


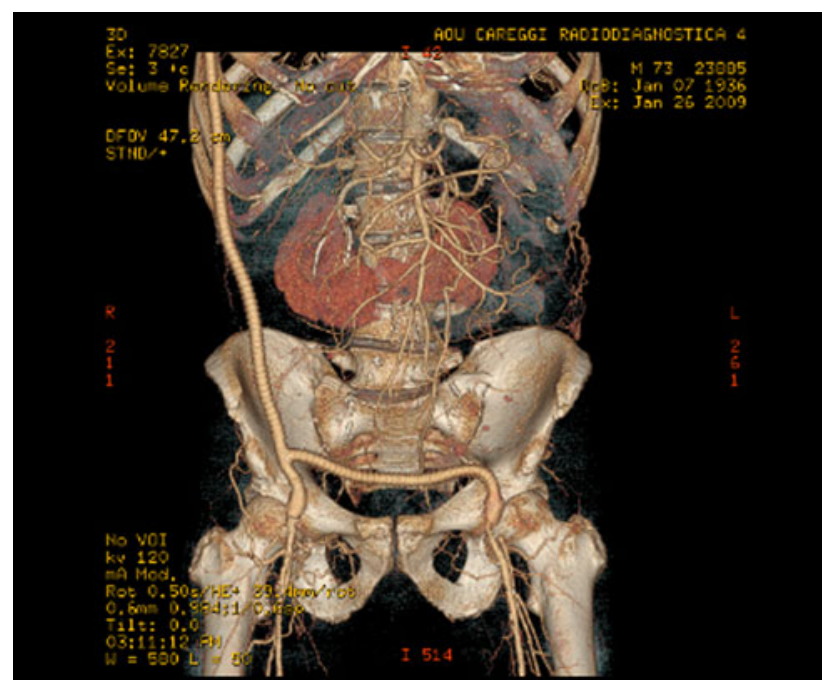

Fig. 3 3D reconstruction from 2D CT scan of the chest and abdomen that shows the axillo-bifemoral bypass graft

gradually, and the patient was extubated. After 1 week, the ICD was reimplanted.

\section{Clinical diagnosis}

Dr. Del Pace: Aortoenteric fistula with periprosthetic infection and possibly secondary $S$. intermedius endocarditis located on the pacemaker catheter.

\section{Discussion}

Prof. Gensini, Dr. Acquafresca: Primary aortoenteric fistulas may occur in case of abdominal aortic aneurysm, infectious aortitis, penetrating peptic ulcer, tumor invasion and radiation therapy [1]. Secondary aortoenteric fistula occurs as a complication of abdominal aortic aneurysm (AAA) repair with an incidence of $0.4-2.4 \%$ [2]. It can occur from 2 weeks to 10 years postsurgery (mean of 32 months) [3]. The most common type of aortoenteric fistula is a communication between the aorta and the third part of duodenum (75\%). Fistulas can also occur in the jejunum, terminal ileum and transverse colon. Infection is ubiquitous in aortoenteric fistulas. Fistulization can be considered a natural although catastrophic extension of perigraft infection [4]. In our case, a secondary infection of the prosthesis by $S$. intermedius from ICD cannot be ruled out. However, there are no data in the literature describing aortic graft involvement as a secondary location of a pacemaker infection, and in this case, cultures from the removed prosthesis were positive only for Gram-negative bacteria.
Reasons leading to fistula formation remain unclear. A hypothesis is that local infection (which can be secondary to the grafting procedure or even pre-existent) might result in intestinal necrosis and a formation of a fistula between the vessel and the intestinal wall [5]. Identification of this complication is one of the most challenging dilemmas in vascular surgery, and in the presence of anemia and fever should be aggressively sought. These two of the most common diagnostic signs, are nonspecific and common in many other disorders. Other frequent symptoms are abdominal or back pain, fatigue, loss of weight, acute or chronic gastrointestinal bleeding, a palpable or a pulsatile abdominal mass $[6,7]$. An early diagnosis is mandatory in order to prevent disastrous complications. A retrospective study conducted on 55 patients who underwent aortic reconstructive surgery with graft placement for treatment of abdominal aortic aneurysm or aortoiliac occlusive disease, demonstrates perioperative and late mortality rates of 17 and $45 \%$, respectively [4].

The CT scan is considered the imaging investigation of choice in suspected aortoenteric fistula [8], even if some authors suggest gastroenteric endoscopy as the initial diagnostic procedure to perform [7].

In a study of Huges et al., six CT signs of the disease are described (sensitivity and specificity are shown in Table 2). Perigraft hematoma is a normal finding after a postoperative period of 3 months, and retained ambient gas is a normal finding for a postoperative period of 2 weeks (after that, any amount of perigraft soft tissue, fluid, or ectopic gas should be considered signs of graft infection).

A negative scan in the presence a suggestive clinical picture does not rule out the diagnosis, but mandates further investigations [9]. Some authors recommend the omission of oral contrast with the abdominal CT scan in order to demonstrate contrast extravasation from the aorta into the bowel lumen [10]. In case of a negative CT scan, additional studies-including magnetic resonance imaging (MRI), gastroenteric endoscopy, In-labeled white blood

Table 2 CT findings in aortoenteric fistulas [9]

\begin{tabular}{lllll}
\hline CT sign & $\begin{array}{l}\text { AEF } \\
\text { cases } \\
(n=10)\end{array}$ & $\begin{array}{l}\text { Control } \\
\text { cases } \\
(n=12)\end{array}$ & $\begin{array}{l}\text { Sensitivity } \\
(\%)\end{array}$ & $\begin{array}{l}\text { Specificity } \\
(\%)\end{array}$ \\
\hline $\begin{array}{l}\text { Periaortic fluid/ } \\
\quad \text { soft tissue }\end{array}$ & 9 & 1 & 90 & 92 \\
Breach in aortic wall & 8 & 3 & 80 & 75 \\
Pseudoaneurysm & 4 & 1 & 40 & 92 \\
Loss of fat pad & 9 & 8 & 90 & 33 \\
Ectopic gas & 4 & 0 & 40 & 100 \\
$\quad \begin{array}{l}\text { Intravasation of } \\
\text { contrast into lumen }\end{array}$ & 0 & 0 & 0 & 100 \\
\hline
\end{tabular}

$A E F$ aortoenteric fistula 
cell scanning, red blood cell scintigraphy, positron emission tomography - are alternative diagnostic examinations that can be utilized.

The sensitivity of MRI has not been investigated, and its diagnostic value is unclear $[8,11]$. Sensitivity of upper GI endoscopy has been reported to be $50-55 \%[7,12]$, and it is reported to be useful mainly for excluding other causes of bleeding [13, 14]. Studies of aortic angiography report diagnostic yields of less than $10 \%[15,16]$. Many studies demonstrate PET usefulness in detecting periprosthetic infection, even if controlled studies are needed to assess its actual utility [14, 17]. Inability to define the anatomic location of tracer activity is not an irrelevant PET limit. Furthermore, visual side-by-side review of PET and a CT scan performed separately is not accurate enough to locate the PET localization in the precise anatomic site visible on
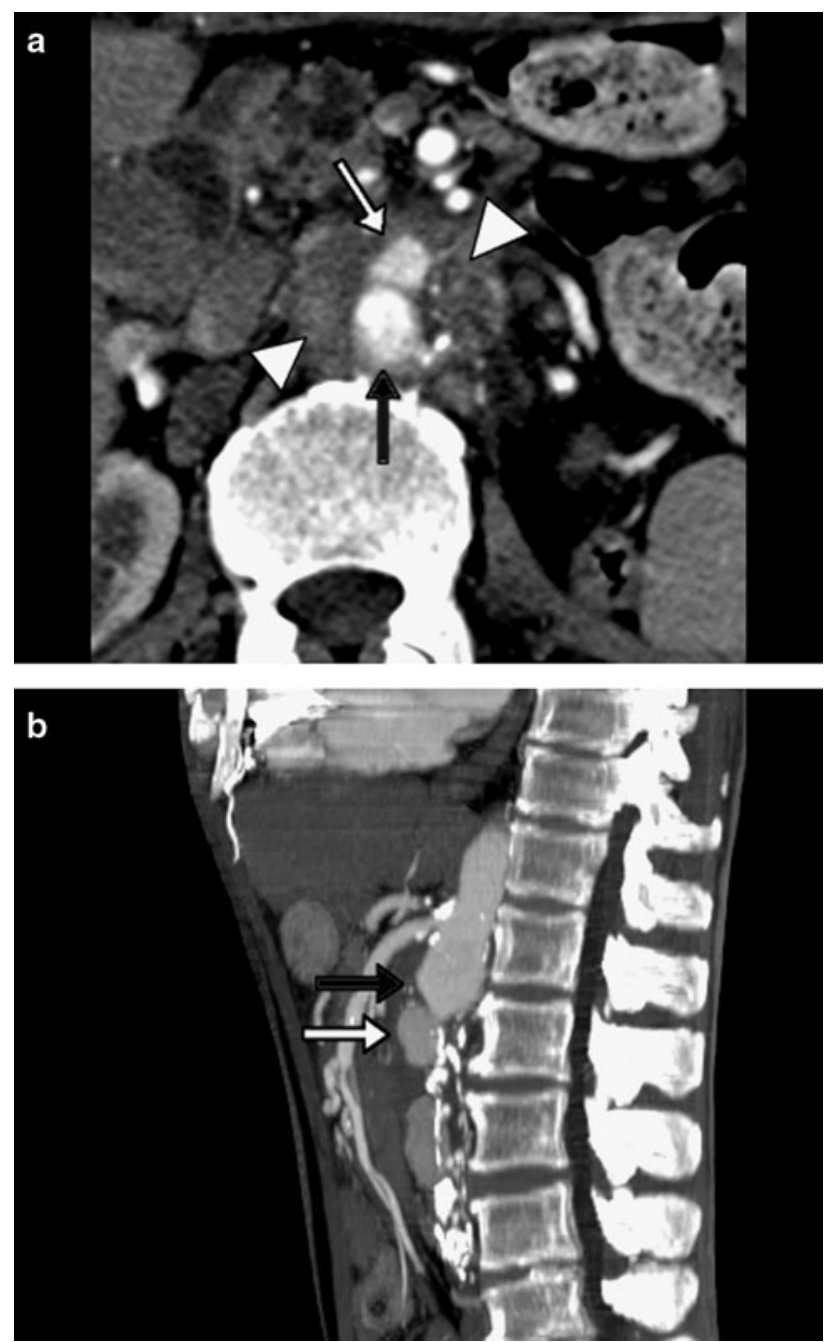

Fig. 4 Pseudoaneurysm of the abdominal aorta in a patient who had undergone aortobifemoral bypass removal. This CT scan shows the native aorta (black arrow), pseudoaneurysm (white arrow), and the periaortic hematoma (arrowhead) the CT scan. On the other hand, a recent study reveals a specificity of $91 \%$ for the combined PET/CT in detecting vascular graft infection, a novel useful tool for the noninvasive diagnosis of the disease [18].

\section{Follow-up}

Dr. Savino: The patient felt quite well 3 months after discharge, with increased hemoglobin levels $(13 \mathrm{~g} / \mathrm{dL})$. He started walking again without leg pain bilaterally. A repeat CT scan of the abdomen, performed 1 month after discharge, showed regular canalization of the axillo-bifemoral graft, and no fluid collection in the site of previous graft.

Twenty months after discharge, the patient complained of acute severe low back pain. A CT scan of the abdomen with the administration of contrast material demonstrated ectasia of the aorta above the stenotic tract, thickened walls, and a periaortic hematoma, suggesting an aortic wall inflammation with the involvement of the small bowel. The day after, the patient had an episode of loss of consciousness, with trismus, rectal bleeding, and severe hypotension despite liquids and supportive therapy. A new CT scan of the abdomen with the administration of contrast material revealed the rupture of the abdominal aorta with fistulization into the small bowel with a massive enteric bleeding (Fig. 4a, b). The patient died later the same day. An autopsy study was not performed.

Conflict of interest None.

\section{References}

1. Nagy SW, Marshall JB (1993) Aortoenteric fistulas. Recognizing a potentially catastrophic cause of gastrointestinal bleeding. Postgrad Med 93:211-219

2. Bertges D, Villella E (2003) Aortoenteric fistula due to endoleak coil embolisation after endovascular AAA repair. J Endovasc Ther 10:130-135

3. Bastounis E, Papalambros E (1997) Secondary aortoduodenal fistulae. J Cardiovasc Surg 38:457-464

4. Low RN, Wall SD (1990) Aortoenteric fistula and perigraft infection: evaluation with CT. Radiology 175:157-162

5. Ueno M, Iguro Y (2006) Aortoenteric fistula after endovascular stent grafting for an abdominal aortic aneurysm report of a case. Surg Today 36:548-564

6. Reily LM, Altman H (1984) Late results following surgical management of vascular graft infection. J Vasc Surg 1:36-44

7. Soetevent C, Klemm PL (2004) Vascular graft infection in aortoiliac and aortofemoral bypass surgery: clinical presentation, diagnostic strategies and results of surgical treatment. Neth J Med $11: 446-452$

8. Mylona S, Ntai S (2007) Aorto-enteric fistula: CT findings. Abdom Imaging 32:393-397

9. Hughes FM, Kavanagh D (2007) Aortoenteric fistula: a diagnostic dilemma. Abdom Imaging 32:398-402 
10. Puvaneswary M, Cuganesan R (2003) Detection of aortoenteric fistula with helical CT. Australas Radiol 47:67-69

11. Orton DF, LeVeen RF (2000) Aortic prosthetic graft infections: radiologic manifestations and implications for management. Radiographics 20:977-993

12. Flye MW, Thompson WM (1983) Aortic graft-enteric and para prosthetic-enteric fistula. Am J Surg 146:183-187

13. Brims FJ, Edwards DP (1999) Gastrointestinal hemorrhage after abdominal aortic grafting. J R Soc Med 92:307-309

14. Krupnik AS, Lombardi JV (2003) 18-Fluorodeoxyglucose positron emission tomography as a novel imaging tool for the diagnosis of aortoenteric fistula and aortic graft infection: a case report. Vasc Endovasc Surg 37:363-366

15. Mark AS, Moss AA (1985) CT of aortoenteric fistulas. Invest Radiol 20:272-275

16. Kleinman LH, Towne JB (1979) A diagnostic and therapeutic approach to aortoenteric fistulas: clinical experience with 20 patients. Surgery $86: 868-880$

17. Stadler P, Bilohlavek O (2004) Diagnosis of vascular prosthesis infection with FDG-PET/CT. J Vasc Surg 40:1246-1247

18. Keidar Z, Engel A (2007) Prosthetic vascular graft infection: the role of 18F-FDG-PET. J Nucl Med 8:1230-1236 